\title{
APPROXIMATION DES FONCTIONS LISSES SUR CERTAINES LAMINATIONS
}

\author{
ROMAIN DUJARDIN
}

\begin{abstract}
RÉSUMÉ. Nous montrons que sur une lamination par surfaces de Riemann localement plongée dans $\mathbb{C}^{2}$, les fonctions $C^{1}$ sont localement limites uniformes de fonctions $C^{1}$ de l'espace ambiant, avec contrôle $L^{p}$ des dérivées le long des feuilles. On en déduit qu'un courant dominé par un courant uniformément laminaire dans $\mathbb{C}^{2}$ est uniformément laminaire.
\end{abstract}

\section{INTRODUCTION}

Les laminations par surfaces de Riemann sont des objets devenus usuels en dynamique holomorphe uni- et multi-dimensionnelle. En dimension complexe 2, toute réunion de graphes disjoints dans un bidisque forme une telle lamination, souvent appelée mouvement holomorphe.

Il est bien connu que l'holonomie d'un mouvement holomorphe n'est en général pas lisse, ce qui est source de difficultés. Le problème se pose en particulier pour la détermination de la structure locale des "courants structuraux" (Sullivan [Su, voir en particulier la note p.236) ou "courants dirigés" (Berndtsson-Sibony [BS] et Fornæss-Sibony [FS]) par une telle lamination.

Nous montrons ici le théorème de régularité suivant pour une lamination par surfaces de Riemann plongée dans un ouvert $\Omega \subset \mathbb{C}^{2}$ (Théorème 2.3). On consultera le paragraphe 2.1] pour la notion de fonction $C^{1}$ sur une lamination.

Théorème 1. Toute fonction de classe $C^{1}$ sur une telle lamination est limite uniforme de restrictions de fonctions $C^{1}$ de l'espace ambiant, avec controle $L^{p}$ des dérivées le long des feuilles.

Il est à noter que ce résultat ne résulte pas directement des théorèmes usuels de régularité des homéomorphismes quasiconformes, qui ne traitent que des dérivées transverses aux feuilles (en particulier nous ne savons pas étendre notre théorème au cas des laminations par hypersurfaces dans $\left.\mathbb{C}^{n}\right)$.

Le théorème a une conséquence sur la structure locale des courants laminaires (Théorème 1.1) qui était à l'origine de ce travail. Nous obtenons en particulier le résultat suivant (Corollaire 1.2) :

Théorème 2. Si $T$ est un courant uniformément laminaire dans $\Omega \subset \mathbb{C}^{2}$ et $S$ est un courant positif fermé tel que $S \leq T$, alors $S$ est uniformément laminaire.

Une motivation pour l'étude de la régularité des laminations par surfaces de Riemann en dimension 2, et des courants qu'elles supportent, est la question suivante, qui demeure toujours ouverte :

Date: le 11 février 2005.

Codes Classif. AMS: 53C12, 30C62. 
Existe t'il une lamination par surfaces de Riemann dans $\mathbb{P}^{2}$ munie d'une mesure transverse invariante?

La réponse (négative) à cette question a été donnée dans des cas particuliers :

- par Camacho-Lins Neto-Sad [CLS] (voir également Deroin De]) dans le cas où la lamination est subordonnée à un feuilletage holomorphe singulier. Ces auteurs utilisent la construction de Bott d'une connexion plate dans le fibré normal à la lamination, qui doit pour ceci être de classe $C^{1}$.

- Fornæss-Sibony [FS démontrent un résultat de non existence de telles laminations en supposant soit une hypothèse de régularité transverse sur la lamination (voir également [HM dans ce cas), soit une hypothèse de régularité sur la mesure transverse. Ceci leur permet essentiellement de considérer le produit $T \wedge T$, où $T$ est le cycle feuilleté associé, pour parvenir à une contradiction.

Dans les deux cas, c'est un problème de régularité de l'holonomie qui empêche l'extension au cas général. Les difficultés soulevées sont du même ordre que celles que nous rencontrerons dans cet article.

\section{Mouvements holomorphes et COURAnts}

1.1. Mouvements holomorphes. Dans tout l'article, le cadre d'étude sera le suivant : on se donne une famille $\mathcal{L}$ de graphes $L_{\alpha}=\left\{w=\varphi_{\alpha}(z)\right\}$ au dessus du disque unité $\mathbb{D}$ dans $\mathbb{C}^{2}$, indexée par un paramètre transverse $\alpha \in \tau$, où $\tau \subset\{0\} \times \mathbb{D}$. On supposera de plus que pour tout $\alpha \in \tau$ et tout $z \in \mathbb{D},\left|\varphi_{\alpha}(z)\right| \leq 1-\varepsilon<1$. En passant à la clôture de la famille de graphes, on pourra supposer que $\tau$ est fermée.

Dans un article classique, Mañé, Sad et Sullivan [MSS] ont montré que l'application d'holonomie

$$
h_{z, z^{\prime}}:(\{z\} \times \mathbb{D}) \cap \mathcal{L} \rightarrow\left(\left\{z^{\prime}\right\} \times \mathbb{D}\right) \cap \mathcal{L}
$$

est continue et quasiconforme. Une telle application est en général appelée mouvement holomorphe. La famille de graphes $\mathcal{L}$ admet ainsi une structure de lamination par surfaces de Riemann. Concluant une longue série de travaux, Słodkowski [St] a démontré qu'un mouvement holomorphe défini sur $\tau$ admet toujours une extension à un mouvement holomorphe de $\mathbb{C}$ tout entier. La preuve à notre avis la plus accessible de ce théorème est celle de Chirka $\mathbb{C}$.

Après extension, l'holonomie est définie dans $\mathbb{C}$ et quasiconforme, elle vérifie donc une équation de Beltrami

$$
\frac{\partial h_{0, z}}{\partial \bar{w}}=\mu^{z}(w) \frac{\partial h_{0, z}}{\partial w}, \quad\left|\mu^{z}(w)\right|<1 .
$$

Il est connu que $z \mapsto \mu^{z}(w)$ est holomorphe, et donc d'après le lemme de Schwarz, $\left|\mu^{z}\right| \leq|z|$. En particulier le coefficient de Beltrami $\mu^{z}$ est arbitrairement petit si le temps de vie du mouvement holomorphe est suffisamment court.

Une conséquence est que l'application d'holonomie est Höldérienne. Il est possible de donner une preuve directe du caractère Hölder de l'holonomie de la façon suivante : si $\alpha, \beta \in \tau$ sont distincts, la fonction

$$
k: s \mapsto-\log \frac{1}{2}\left|\varphi_{\alpha}(s)-\varphi_{\beta}(s)\right|
$$

est harmonique et strictement positive. De l'inégalité de Harnack

$$
\frac{1-|z|}{1+|z|} \leq \frac{k(z)}{k(0)} \leq \frac{1+|z|}{1-|z|}
$$


on déduit que

$$
2^{\frac{-2|z|}{1-|z|}}\left|\varphi_{\alpha}(0)-\varphi_{\beta}(0)\right|^{\frac{1+|z|}{1-|z|}} \leq\left|\varphi_{\alpha}(z)-\varphi_{\beta}(z)\right| \leq 2^{\frac{2|z|}{1+|z|}}\left|\varphi_{\alpha}(0)-\varphi_{\beta}(0)\right|^{\frac{1-|z|}{1+|z|}} .
$$

Un corollaire est que la constante de Hölder peut être choisie arbitrairement proche de 1, quitte à réduire le disque de base.

1.2. Courants laminaires et dirigés. Si $\mu$ est une mesure positive finie sur $\tau$, la formule

$$
T=\int_{\tau}\left[L_{\alpha}\right] d \mu(\alpha)
$$

définit un courant positif fermé de masse localement finie dans $\mathbb{D}^{2}$ qui est dit uniformément laminaire et subordonné à la lamination $\mathcal{L}$.

On peut définir une autre classe de courants associés à $\mathcal{L}$. Sullivan [Su définit les courants structuraux -que suivant Sibony, nous appellerons dirigés- comme le cône convexe fermé engendré par les courants de la forme $i \delta_{p}(d \ell(p) \wedge d \bar{\ell}(p))$ où $d \ell(p)$ est une forme $\mathbb{C}$-linéaire dont le noyau est $T_{p} L$, où $L$ est une feuille de $\mathcal{L}$ et $p \in L$. Dans la situation ci-dessus, on peut normaliser le choix de $d \ell$ en imposant que $d \ell(0,1)=1$; alors si $p \in L_{\alpha}, d \ell(p)=d w-\varphi_{\alpha}^{\prime}(z) d z$. On vérifie aisément que la $(1,0)$ forme $d \ell$ est continue, et un courant positif est dirigé par $\mathcal{L}$ ssi $T \wedge d \ell=0$. Il est clair qu'un courant uniformément laminaire est dirigé. Plus généralement, tout courant de la forme $f T$, où $T$ est uniformément laminaire et $f \in L_{l o c}^{1}(\|T\|)(\|T\|$ désigne la mesure trace de $T$ ) est dirigé.

La question que nous nous posons est alors la suivante : est il vrai que tout courant positif fermé dirigé par un mouvement holomorphe $\mathcal{L}$ est uniformément laminaire? Si $\mathcal{L}$ est de classe $C^{1}$, le résultat se trouve dans $[\mathrm{Su}$. Dans le cas général nous n'avons que le résultat partiel suivant.

Théorème 1.1. Soit $T$ un courant uniformément laminaire subordonné à une lamination par graphes au dessus de $\mathbb{D}$. Alors tout courant positif fermé de la forme $f T$, où $f \in L_{l o c}^{1+\varepsilon}(\|T\|)$, $\varepsilon>0$, est uniformément laminaire.

Corollaire 1.2. Si T est uniformément laminaire et $S$ est un courant positif fermé tel que $S \leq T$, alors $S$ est uniformément laminaire.

Démonstration. En effet d'après le théorème de Radon-Nikodym il existe une fonction mesurable $0 \leq f \leq 1$ telle que $\|S\|=f\|T\|$. Par ailleurs le courant $T$ admet une décomposition polaire

$$
T=\int\langle t(p), \cdot\rangle d\|T\|(p),
$$

où $t(p)$ est un $(1,1)$ vecteur positif de norme 1 , défini $\|T\|$ presque partout. $T$ étant uniformément laminaire, le vecteur $t(p)$ est presque partout simple, i.e. de la forme $i e \wedge \bar{e}$. On en déduit aisément que $S=\int\langle t(p), \cdot\rangle f d\|T\|(p)=f T$.

Remarque 1.3. Le résultat étant de nature locale, le théorème vaut également pour un courant subordonné à une lamination plongée dans une surface complexe.

La preuve du théorème 1.1 que nous présentons maintenant est en quelque sorte une version rigoureuse du raisonnement heuristique suivant

$$
d(f T)=0 \Longrightarrow f \text { constante le long des feuilles. }
$$

Elle repose de manière cruciale sur le théorème d'approximation 2.3. 
Preuve du théorème. On pose $S=f T$. Soit $\chi$ une fonction continue sur la transversale $\tau$. On peut prolonger $\chi$ en une fonction, toujours notée $\chi$, constante le long des feuilles.

Lemme 1.4. Le courant $\chi S$ est fermé.

Démonstration. Soit $\phi$ une 1-forme test. On veut montrer que $\langle\chi S, d \phi\rangle=0$. Supposons pour un instant que l'holonomie soit lisse de classe $C^{1}$. Dans ce cas on peut écrire

$$
\langle\chi S, d \phi\rangle=-\langle S, d \chi \wedge \phi\rangle=\int_{\tau}\left(\int_{L_{\alpha}} f d \chi \wedge \phi\right) d \mu(\alpha)=0
$$

car $\chi$ est $C^{1}$ et constante le long des feuilles.

Dans le cas général la fonction $\chi$ n'est plus $C^{1}$, et les calculs précédents n'ont plus de sens. D'après le théorème 2.3, pour tout $1 \leq p<\infty \chi$ est limite uniforme d'une suite de fonctions lisses $\chi_{n}$ de sorte que les dérivées de $\chi_{n}$ le long de $L_{\alpha}$ tendent vers 0 dans $L^{p}$, uniformément en $\alpha$. On choisit $p>\frac{1+\varepsilon}{\varepsilon}$, et on écrit alors

$$
\langle\chi S, d \phi\rangle=\lim _{n \rightarrow \infty}\left\langle\chi_{n} S, d \phi\right\rangle=\int_{\tau}\left(\int_{L_{\alpha}} f d \chi_{n} \wedge \phi\right) d \mu(\alpha) .
$$

Ce dernier terme tend vers 0 car $f \in L^{1+\varepsilon}$ et $p>\frac{1+\varepsilon}{\varepsilon}$.

Le théorème est alors une conséquence directe de la caractérisation suivante des courants uniformément laminaires.

Lemme 1.5. Soit $S$ un courant positif fermé porté par $\mathcal{L}$, tel que pour toute fonction $\chi$ continue et constante le long des feuilles, $\chi S$ soit fermé. Alors $S$ est uniformément laminaire.

Démonstration. On veut montrer que le courant $S$ est dans le cône convexe fermé engendré par les $\left[L_{\alpha}\right]$. Supposons le contraire. D'après le théorème de Hahn-Banach, il existe une forme test $\phi$ telle que $\left\langle\left[L_{\alpha}\right], \phi\right\rangle<0$ pour tout $\alpha$ et $\langle S, \phi\rangle>0$. On considère alors un recouvrement fini de $\tau$ par des ouverts $U_{i}$ de diamètre inférieur à $\frac{1}{10}$, et une partition continue de l'unité $\theta_{i}$ associée. On prolonge les fonctions $\theta_{i}$ de manière constante le long des feuilles. D'après le lemme, chacun des courants $\theta_{i} S$ est fermé. De plus

$$
\langle S, \phi\rangle=\sum\left\langle\theta_{i} S, \phi\right\rangle>0
$$

donc il existe $i$ tel que $\left\langle\theta_{i} S, \phi\right\rangle>0$. On pose $S_{1}=c_{i} \theta_{i} S$, où la constante $c_{i}>0$ est telle que $\mathbf{M}\left(S_{1}\right)=1$. Comme $S_{1}=c_{i} \theta_{i} f T, S_{1}$ est de la forme $f_{1} T$, où $f_{1} \in L_{l o c}^{1+\varepsilon}(\|T\|)$.

On refait la même construction pour $S_{1}$, avec des ouverts de diamètre $<\frac{1}{100}$. En appliquant itérativement le procédé, on obtient une suite de courants $S_{k}$ positifs fermés de masse 1, dont les supports convergent vers une certaine feuille $L$. Ainsi $S_{k} \rightarrow c[L]$, avec $c>0$. Mais $\left\langle S_{k}, \phi\right\rangle>0$ alors que $\langle[L], \phi\rangle<0$, ce qui est contradictoire.

Remarque 1.6. Le lemme 1.5] a pour conséquence directe un résultat dû à N. Sibony [Si] : si $\mathcal{L}$ est une lamination transversalement totalement discontinue, et $T$ est un courant positif fermé porté par $\mathcal{L}$, alors $T$ est uniformément laminaire.

\section{LE THÉORÈME D’APPROXIMATION}

2.1. Structure $C^{1}$. Rappelons d'abord ce qu'on entend par fonction de classe $C^{1}$ sur une lamination. Une fonction $f$ sur $\mathcal{L}$ sera dite de classe $C^{1}(\mathcal{L})$ si elle est $C^{1}$ le long des feuilles et que les dérivées de $f$ le long des feuilles sont transversalement continues. On norme l'espace $C^{1}(\mathcal{L})$ de manière naturelle. 
Il convient de remarquer que même si $\mathcal{L}$ est un feuilletage de classe $C^{1}$, une fonction $C^{1}(\mathcal{L})$ n'est pas nécessairement la restriction d'une fonction $C^{1}$ de l'espace ambiant.

On a néanmoins dans ce cas un résultat d'approximation [De, Lemme 3.23], dont nous incluons une preuve succinte pour la commodité du lecteur.

Lemme 2.1. Si $\mathcal{L}$ est de classe $C^{1}$, toute fonction $f \in C^{1}(\mathcal{L})$ est limite dans $C^{1}(\mathcal{L})$ de restrictions de fonctions $C^{1}$ de l'espace ambiant.

Démonstration. Après redressement par un difféomorphisme de classe $C^{1}$, on peut supposer que $\mathcal{L}$ est la lamination naturelle de $\mathbb{D} \times \tau$. Si $g$ est une fonction continue sur $\tau$, on dispose d'un prolongement continu $\mathcal{E}(g)$ de $g$ à $\mathbb{C}$, où $\mathcal{E}$ est l'opérateur de prolongement de Whitney $C(\tau) \rightarrow C(\mathbb{C})$ (voir $[\mathrm{St}]$ ). En particulier la fonction

$$
(z, \alpha) \mapsto \mathcal{E}(f(z, \cdot))(\alpha)
$$

est définie dans $\mathbb{D} \times \mathbb{C}$. Comme $\mathcal{E}$ est linéaire et continu, le prolongement est $C^{1}$ par rapport à la variable $z$. Il ne reste qu'à convoler par un noyau régularisant de variable $\alpha$ pour obtenir l'approximation voulue.

Remarque 2.2. Par un argument analogue à la preuve du Théorème 1.1 on montre que si $\mathcal{L}$ est une lamination par surfaces de Riemann plongée satisfaisant la conclusion du lemme, tout courant positif fermé dirigé par $\mathcal{L}$ est uniformément laminaire (voir également $\mathrm{De}$, Proposition $3.22])$.

2.2. Approximation. La notation $\mathcal{L}$ désigne toujours une lamination par graphes au dessus du disque unité. On écrira $L \in \mathcal{L}$ pour dire que $L$ est une feuille (plaque) de $\mathcal{L}$, et $\nabla_{L}$ désigne le gradient le long de $L$. Pour $p>1$ on désigne par $W^{1, p}(\mathcal{L})$ l'espace de "Sobolev" des fonctions continues sur $\mathcal{L}$ dont les dérivées dans les feuilles sont dans $L^{p}$, et varient continûment au sens où l'application $L \mapsto \nabla_{L} f$ est continue. On le munit de la norme

$$
\|f\|_{W^{1, p}}=\|f\|_{L^{\infty}}+\sup _{L \in \mathcal{L}}\left\|\nabla_{L} f\right\|_{L^{p}}
$$

qui en fait un espace de Banach.

Le théorème d'approximation est le suivant.

Théorème 2.3. Soit $1<p<\infty$ et $f \in C^{1}(\mathcal{L})$. Il existe une suite $\left(f_{n}\right)$ de fonctions lisses dans $\mathbb{D} \times \mathbb{C}$ telles que

$$
\left\|f-f_{n}\right\|_{W^{1, p}} \underset{n \rightarrow \infty}{\longrightarrow} 0
$$

sur tout compact.

La preuve sera découpée en étapes et occupera le reste de la section.

Localisation. Remarquons d'abord qu'en recollant des approximations locales avec une partition de l'unité, on peut se contenter de résoudre le problème dans un voisinage arbitraire $\{0\} \times D(0, r)$ de $\{0\} \times \mathbb{D}$. En particulier le coefficient de dilatation de l'équation de Beltrami (11) ci-dessous pourra être choisi arbitrairement petit. On renormalise ensuite pour considérer à nouveau une lamination dans $\mathbb{D} \times \mathbb{C}$.

Extension. Grâce au théorème de Słodkowski, on étend la lamination, toujours notée $\mathcal{L}$, à $\mathbb{D} \times \mathbb{C}$. On peut supposer que les feuilles sont des droites horizontales hors du bidisque 
unité. Comme au 1.1, l'holonomie entre droites verticales vérifie maintenant une équation de Beltrami

$$
\frac{\partial h_{z, z^{\prime}}}{\partial \bar{w}}=\mu^{z, z^{\prime}}(w) \frac{\partial h_{z, z^{\prime}}}{\partial w}, \quad\left|\mu^{z, z^{\prime}}(w)\right| \leq \kappa=\frac{2 r}{1+r^{2}}<1,
$$

où $r$ été défini ci-dessus. L'application d'holonomie $h^{z, z^{\prime}}$ est définie dans $\{z\} \times \mathbb{C}$, et par la suite on écrira indistinctement $h^{z, z^{\prime}}(z, w)$ et $h^{z, z^{\prime}}(w)$. Remarquons que $\mu^{z, z^{\prime}}$ est défini dans $\mathbb{C}$, et s'annule identiquement hors de $\mathbb{D}$.

Une première approximation. Sur $\mathcal{L}$ on dispose de deux systèmes de coordonnées : les coordonnées standard $(z, w)$ dans $\mathbb{D} \times \mathbb{C}$ et les coordonnées $(z, \alpha)$, où $\alpha \in \mathbb{C} \simeq\{0\} \times \mathbb{C}$ indexe la feuille $L_{\alpha}$ à laquelle le point courant $(z, w)$ appartient.

L'application

$$
\Phi:(z, w) \longmapsto(z, \alpha)=\left(z, h^{z, 0}(z, w)\right)
$$

est un homéomorphisme qui redresse $\mathcal{L}$, et qui est de classe $C^{1}(\mathcal{L})$. La fonction $f \circ \Phi^{-1}$ est définie sur le fermé $\mathbb{D} \times \tau$ de la lamination redressée. En utilisant le Lemme 2.1 on approche $f \circ \Phi^{-1}$ par des fonctions $g_{n}$, de classe $C^{1}$ (au sens usuel) dans $\mathbb{D} \times \mathbb{C}$ pour la topologie $C^{1}$ de la lamination redressée. On a maintenant $g_{n} \circ \Phi \rightarrow f$ dans $W^{1, p}(\mathcal{L})$, et les fonctions $g_{n}=f_{n} \circ \Phi^{-1}$ sont $C^{1}$ par rapport à la variable $(z, \alpha)$ (mais pas par rapport à $(z, w)$ ). Quitte à remplacer $f$ par un $f_{n}$ assez proche, il suffit donc de démontrer le théorème quand $f \circ \Phi^{-1}(z, \alpha)$ est $C^{1}$ par rapport à $(z, \alpha){ }^{1}$

Convolution. On convole le coefficient de Beltrami $\mu^{z}(w)=\mu^{0, z}(w)$ par un noyau régularisant $\theta_{\varepsilon}(w)$. Le nouveau noyau $\mu_{\varepsilon}^{z}(w)=\mu^{z}(w) * \theta_{\varepsilon}(w)$ est toujours holomorphe en $z$ donc l'unique solution $h_{\varepsilon}^{0, z}(w)$ injective et tangente à l'identité à l'infini de l'équation de Beltrami correspondante est l'holonomie d'une lamination par graphes $\mathcal{L}_{\varepsilon}$ dans $\mathbb{D} \times \mathbb{C}$. D'après un théorème classique (voir Ahlfors $[\mathrm{A}]$ ), $w \mapsto h_{\varepsilon}^{0, z}(w)$ est de classe $C^{1}$, et quand $\varepsilon \rightarrow 0, h_{\varepsilon}^{0, z}$ converge uniformément vers $h^{0, z}$, et les dérivées de $h_{\varepsilon}^{0, z}$ (par rapport à $w$ ) convergent en norme $L^{p(\kappa)}$. L'exposant $p(\kappa)$ tend vers l'infini quand $\kappa \rightarrow 0$. On choisit $\kappa$ de sorte que $p(\kappa)>p, p$ fixé par l'énoncé du théorème.

La famille de laminations $\mathcal{L}_{\varepsilon}$ tend vers $\mathcal{L}$ au sens où la feuille $L_{\varepsilon}(\alpha)$ issue de $(0, \alpha)$ converge vers $L_{\alpha}$, uniformément relativement à $\alpha$. Soit $\Phi_{\varepsilon}$ l'homéomorphisme de redressement de $\mathcal{L}_{\varepsilon}$ comme précédemment. On définit la fonction $f_{\varepsilon}$ par

$$
f_{\varepsilon}(z, w)=f \circ \Phi^{-1} \circ \Phi_{\varepsilon}(z, w) \Leftrightarrow f_{\varepsilon} \circ \Phi_{\varepsilon}^{-1}(z, \alpha)=f \circ \Phi^{-1}(z, \alpha),
$$

autrement dit après redressement, " $f_{\varepsilon}=f$ ". Il est clair que $f_{\varepsilon}$ tend uniformément vers $f$ quand $\varepsilon \rightarrow 0$. Nous allons montrer que cette approximation résout notre problème.

$f_{\varepsilon}$ est $C^{1}$. Il faut prendre garde au fait qu'une lamination dont l'holonomie est $C^{1}$ n'est pas un feuilletage $C^{1}$ en général (voir le très éclairant $\S 6$ de [PSW]). Ici, nous allons vérifier que l'application de carte

$$
\Phi_{\varepsilon}^{-1}:(z, \alpha) \mapsto(z, w)=\left(z,\left(h_{\varepsilon}^{0, z}\right)(\alpha)\right)
$$

\footnotetext{
${ }^{1}$ Dans le cas, suffisant pour le théorème 1.1 où $f$ est constante le long des feuilles, l'utilisation du Lemme 2.1 est superflue. Il suffit d'approcher uniformément $f$ par des fonctions lisses dans la transversale $\{0\} \times \mathbb{C}$ et d'étendre les fonctions lisses en constantes le long des feuilles.
} 
est de classe $C^{1}$ par rapport à la variable $(z, \alpha)$. Puisque $h_{\varepsilon}^{0, z}(\alpha)=\varphi_{\alpha}^{\varepsilon}(z)$ est holomorphe en $z$, il suffit de vérifier que les dérivées dans la direction verticale de $h_{\varepsilon}^{0, z}(\alpha)$ sont continues par rapport à $(z, \alpha)$.

L'argument est le suivant (voir [CG pp. 22-24] ou [A]) : soit $\mu_{\varepsilon}^{z}(\alpha)$ le coefficient de Beltrami associé à $h_{\varepsilon}^{0, z}(\alpha) ; \mu_{\varepsilon}^{z}(\alpha)$ est $C^{1}$ en $\alpha$ et holomorphe en $z$. En particulier $\frac{\partial}{\partial \alpha} \mu_{\varepsilon}^{z}(\alpha)$ est continue en $(z, \alpha)$. On peut écrire $\frac{\partial}{\partial \alpha} h_{\varepsilon}^{0, z}(\alpha)=e^{\varphi}$, où $\varphi$ vérifie une équation de type Beltrami de variable $z$, dont le second membre est $\frac{\partial}{\partial \alpha} \mu_{\varepsilon}^{z}(\alpha)$. Ceci implique que les dérivées de $h_{\varepsilon}^{0, z}$ dans la direction verticale sont uniformément bornées et Hölderiennes d'exposant $1-\frac{2}{p}$ en $\alpha$. Par ailleurs $\frac{\partial}{\partial \alpha} h_{\varepsilon}^{0, z}$, vue comme fonction de $\alpha$, varie continûment dans $L^{p}$ relativement à la variable $z$, et donc continûment pour la topologie de la convergence uniforme, par équicontinuité. On en déduit la continuité de $\frac{\partial}{\partial \alpha} h_{\varepsilon}^{0, z}$ en la variable $(z, \alpha)$.

Convergence en norme $W^{1, p}$. On fixe maintenant une feuille $L$ de $\mathcal{L}$, et on va montrer que $\left\|\nabla_{L} f_{\varepsilon}-\nabla_{L} f\right\|_{W^{1, p}} \rightarrow 0$. Le lecteur vérifiera aisément que l'uniformité en $L$ est conséquence de la preuve.

Quitte à redresser par une application holomorphe, on peut toujours supposer que $L=$ $L_{0}=(w=0)$. On pose $\widetilde{f}_{\varepsilon}=f_{\varepsilon} \circ \Phi_{\varepsilon}^{-1}$ (et de même pour $f$ ) de sorte que $\widetilde{f}_{\varepsilon}(z, \alpha)=f_{\varepsilon}(z, w)$. Par définition de $f_{\varepsilon}$, on a

$$
f_{\varepsilon}(z, 0)=\widetilde{f}_{\varepsilon}\left(z, h_{\varepsilon}^{z, 0}(z, 0)\right)=\widetilde{f}\left(z, h_{\varepsilon}^{z, 0}(z, 0)\right),
$$

car $h_{\varepsilon}^{z, 0}(z, 0)$ est le projeté du point $(z, 0)$ sur $\{0\} \times \mathbb{C}$ le long de $\mathcal{L}_{\varepsilon}$. Cette projection n'est certainement pas injective, puisque $\mathcal{L}_{\varepsilon}$ est essentiellement parallèle à $\mathcal{L}$.

Deux cas se présentent : soit $L_{0}$ est également une feuille de $\mathcal{L}_{\varepsilon}$, auquel cas $f_{\varepsilon}(z, 0)=f(z, 0)$ et il n'y a rien à démontrer; dans le cas contraire, les intersections de $L$ et $\mathcal{L}_{\varepsilon}$ sont transverses sauf en au plus un nombre localement fini de points [BLS Lemme 6.4], donc en un point générique de $L, L$ est une transversale holomorphe locale à $\mathcal{L}_{\varepsilon}$.

Le point crucial de la preuve est le lemme suivant. Il est peut être déjà connu des spécialistes, mais nous ne l'avons pas trouvé dans la littérature. Rappelons que pour toute paire de droites verticales $\{z\} \times \mathbb{C}$ et $\left\{z^{\prime}\right\} \times \mathbb{C}$, l'holonomie $h_{\varepsilon}^{z, z^{\prime}}$ est quasiconforme et de dilatation majorée par $\kappa$.

Lemme 2.4. Soient $D_{1}$ et $D_{2}$ deux transversales holomorphes locales à une feuille $L_{\varepsilon}^{0}$ de $\mathcal{L}_{\varepsilon}$. Alors l'holonomie

$$
h^{1,2}: D_{1} \subset U_{1} \longrightarrow U_{2} \subset D_{2}
$$

est quasiconforme de dilatation majorée par $\kappa$.

En d'autres termes, la dilatation ne dépend pas des transversales, pourvu qu'elles soient holomorphes.

Preuve du lemme. La lamination $\mathcal{L}_{\varepsilon}$ est en fait un feuilletage de classe $C^{1}$, donc $h^{1,2}$ est différentiable. Pour simplifier les notations, dans la suite de la preuve nous omettrons les indices $\varepsilon$. Si $L$ est une feuille proche de $L^{0}$ on pose pour $i=1,2, p_{i}=\left(z_{i}, w_{i}\right)=D_{i} \cap L$, de sorte que $h^{1,2}\left(p_{1}\right)=p_{2}$. Les droites $\left\{z_{1}\right\} \times \mathbb{C}$ et $\left\{z_{2}\right\} \times \mathbb{C}$ sont des transversales respectives en $p_{1}$ et $p_{2}$, donc au voisinage de $p_{1}$, on peut décomposer $h^{1,2}$ en $h^{z_{2}, 2} \circ h^{z_{1}, z_{2}} \circ h^{1, z_{1}}$ où $h^{1, z_{1}}$ $\left(\right.$ resp. $\left.h^{z_{2}, 2}\right)$ envoie localement $D_{1}$ sur $\left\{z_{1}\right\} \times \mathbb{C}\left(\operatorname{resp} .\left\{z_{2}\right\} \times \mathbb{C} \operatorname{sur} D_{2}\right)$. 
L'observation ${ }^{2}$ est que $h^{1, z_{1}}$ est différentiable au point $p_{1}$, et sa différentielle est la projection de $T_{p_{1}} D_{1}$ sur $\left\{z_{1}\right\} \times \mathbb{C}$ parallèlement à $T_{p_{1}} L$. C'est une application $\mathbb{C}$-linéaire, et donc son coefficient de Beltrami au point $p_{1}$ est nul. Il en va de même pour $h^{z_{2}, 2}$ en $p_{2}$. Ainsi le coefficient de dilatation de $h^{1,2}$ en $p_{1}$ est majoré par $\kappa$.

Nous pouvons maintenant terminer la preuve du théorème. On veut majorer $\widetilde{f}(z, 0)-$ $\widetilde{f}\left(z, h_{\varepsilon}^{z, 0}(z, 0)\right)$ en norme $W^{1, p}$. D'après la première approximation, $\widetilde{f}$ est $C^{1}$, donc il suffit de montrer que les dérivées de $\pi_{\varepsilon}: z \mapsto h_{\varepsilon}^{z, 0}(z, 0)$ sont petites en norme $L^{p}$.

D'après le lemme, au voisinage de tout point de transversalité de $L=\{w=0\}$ et $\mathcal{L}_{\varepsilon}$, la projection $\pi_{\varepsilon}$ est un homéomorphisme quasiconforme de coefficient de dilatation $\leq \kappa$. Donc hors d'un ensemble discret de points de $L, \pi_{\varepsilon}$ vérifie une équation de Beltrami

$$
\frac{\partial \pi_{\varepsilon}}{\partial \bar{z}}=\nu \frac{\partial \pi_{\varepsilon}}{\partial z}, \text { avec }|\nu| \leq \kappa .
$$

On identifie $L$ et $\mathbb{D}$ et on étend $\nu$ à $\mathbb{C}$ par zéro. Toutes les solutions de cette équation sur $\mathbb{D}$ sont de la forme $\pi_{\varepsilon}=u_{\varepsilon} \circ k_{\varepsilon}$ où $k_{\varepsilon}$ est l'unique homéomorphisme tangent à l'infini solution de (2) et $u_{\varepsilon}$ est holomorphe sur $k_{\varepsilon}(\mathbb{D})$ DB. En particulier $\left\|\nabla k_{\varepsilon}\right\|_{L^{p}} \leq C$ où $C$ ne dépend que de $\kappa$, et les modules de continuité (Hölder) $k_{\varepsilon}$ et $k_{\varepsilon}^{-1}$ ne dépendent également que de $\kappa$.

Par ailleurs, $\mathcal{L}_{\varepsilon}$ est proche de $\mathcal{L}$, donc les feuilles de $\mathcal{L}_{\varepsilon}$ issues de $L$ coupent $\{0\} \times \mathbb{C}$ près de 0 . En particulier l'image de $\pi_{\varepsilon}$, c'est à dire celle de $u_{\varepsilon}$ est de petit diamètre. On fixe un disque légèrement plus petit $D(0,1-\delta) \subset \subset \mathbb{D}$. On a alors l'estimation

$$
\operatorname{dist}\left(\partial\left(k_{\varepsilon}(D(0,1-\delta)), \partial\left(k_{\varepsilon}(\mathbb{D})\right)\right) \geq c(\kappa) \delta^{\theta(\kappa)},\right.
$$

et donc d'après la formule de Cauchy, les dérivées de $u_{\varepsilon}$ sont uniformément petites sur $k_{\varepsilon}(D(0,1-\delta))$. On en conclut que les dérivées de $k_{\varepsilon}=u_{\varepsilon} \circ k_{\varepsilon}$ sont petites en norme $L^{p}$ sur tout compact.

\section{RÉFÉRENCES}

[A] Ahlfors, Lars V. Lectures on quasiconformal mappings. Van Nostrand Co., Inc., Toronto, Ont.-New York-London 1966.

[BLS] Bedford, Eric; Lyubich, Mikhail ; Smillie, John. Polynomial diffeomorphisms of $\mathbb{C}^{2} . I V$ : The measure of maximal entropy and laminar currents. Invent. Math. 112 (1993), 77-125.

[BS] Berndtsson, Bo ; Sibony, Nessim. The $\bar{\partial}$ equation on a positive current. Invent. Math. 147 (2002), 371-428.

[CLS] Camacho, Cesar; Lins Neto, Alcides; Sad, Paulo. Minimal sets of foliations on complex projective spaces. Inst. Hautes Etudes Sci. Publ. Math. No. 68, (1988), 187-203 (1989).

[CG] Carleson, Lennart; Gamelin, Theodore W. Complex dynamics. Universitext : Tracts in Mathematics. Springer-Verlag, New York, 1993.

[C] Chirka, Evgeni M. On the extension of holomorphic motions. Preprint.

[De] Deroin, Bertrand. Laminations par variétés complexes. Thèse, ENS Lyon, 2003.

[DB] Douady, Adrien ; Buff Xavier. Le théorème d'intégrabilité des structures presque complexes. The Mandelbrot set, theme and variations. pp. 307-324. London Math. Soc. Lecture Note Ser., 274, Cambridge Univ. Press, Cambridge 2000.

[FS] Fornæss, John Erik; Sibony, Nessim. Harmonic currents of finite energy and laminations. Preprint (version de janvier 2005).

[HM] Hurder, Steven; Mitsumatsu, Yoshihiko. The intersection product of transverse invariant measures. Indiana Univ. Math. J. 40 (1991), 1169-1183.

\footnotetext{
${ }^{2}$ Ceci reste vrai même si $\mathcal{L}$ n'est pas lisse.
} 
[MSS] Mañé, Ricardo; Sad, Paulo; Sullivan, Dennis On the dynamics of rational maps. Ann. Sci. école Norm. Sup. 16 (1983), 193-217.

[PSW] Pugh, Charles; Shub, Michael ; Wilkinson, Amie. Hölder foliations. Duke Math. J. 86 (1997), 517-546.

[Si] Sibony, Nessim. Communication personnelle.

[St] Słodkowski, Zbigniew. Holomorphic motions and polynomial hulls. Proc. Amer. Math. Soc. 111 (1991), 347-355.

[St] Stein, Elias M. Singular integrals and differentiability properties of functions. Princeton University Press, Princeton, N.J. 1970.

[Su] Sullivan, Dennis. Cycles for the dynamical study of foliated manifolds and complex manifolds. Invent. Math. 36 (1976), 225-255.

UFR de mathématiques, Université Paris 7, Case 7012, 2 Place Jussieu, 75251 Paris CEDEX 05, FRANCE.

dujardin@math.jussieu.fr 